\section{Meet the METs}

\section{By Lauren Martz, Staff Writer}

Researchers at Queen Mary, University of London have found that blocking endocytosis could treat MET-mutant cancers resistant to current treatments. ${ }^{1}$ Developing a safe way to inhibit the essential cellular process could prove challenging.

Endocytosis is a process by which the cell takes up extracellular molecules including proteins for recycling or degradation. The process influences cell migration, survival and receptor signaling. ${ }^{2,3}$

In a paper published in Nature Cell Biology, Stéphanie Kermorgant and colleagues at the Barts Cancer Institute at Queen Mary, University of London have found support for the role of endocytosis in oncogenesis. The team has suggested that mutations in the c-Met proto-oncogene (MET; HGFR) might contribute to oncogenesis not only by inducing aberrant MET activation but also by increasing endocytosis of the MET receptor.

MET is a cell surface receptor tyrosine kinase that is activated in multiple cancers including kidney, liver, breast, stomach and brain. At least 14 inhibitors of the receptor are in clinical testing, including Pfizer Inc.'s crizotinib, a dual MET and anaplastic lymphoma kinase (ALK) inhibitor that is in Phase III testing for non-small cell lung cancer (NSCLC).

Kermorgant's group transfected mouse embryonic fibroblasts with wild-type Met or one of two oncogenic activating Met mutations, D1246N or M1268T. The D1264N mutation was resistant to a Metspecific tyrosine kinase inhibitor, which suggests patients harboring that mutation might not respond to tyrosine kinase inhibition and that factors other than activation of the receptor's tyrosine kinase are contributing to oncogenesis.

In the fibroblasts, mutant Met expression increased both the receptor tyrosine kinase's activation and endocytosis as well as recycling of the receptor from the cell surface. The Met mutant also decreased the receptor's degradation compared with wild-type Met.

The next step was inhibiting Met endocytosis. To do so, the team focused on inhibiting two targets: dynamin (Dnm), a GTPase required for vesicle formation during endocytosis, and the heavy chain of clathrin (Clt), which also is required for the formation of coated vesicles during endocytosis.

Also in mouse fibroblasts, the dynamin inhibitors dynasore and dynole 34-2 blocked the increased cellular migration and growth caused by the mutations compared with vehicle control. The researchers saw similar results with small hairpin RNA or RNAi inhibitors of the clathrin heavy chain.

Endocytosis inhibition did not affect migration or growth of cells expressing wild-type Met.

In mice, tail vein injection of cells expressing the M1268T Met mutant caused lung metastasis and tumor growth compared with injection of cells expressing wild-type Met, which did not cause the formation of lung tumors. In the mice with mutant cells, shRNA against the clathrin heavy chain prevented the establishment of lung tumors compared with control shRNA.

Also in mice, topical dynasore or dynole 34-2 decreased the size of subcutaneous tumors expressing the M1268T or D1264N mutations compared with vehicle control. Similar to the in vitro findings, the Met tyrosine kinase inhibitor was only effective against tumors formed by the M1268T mutation. These findings indicate inhibiting MET endocytosis could have more comprehensive anticancer activity than inhibiting MET tyrosine kinase activity.

"Our study represents a proof of principle that manipulating the localization of an oncogenic receptor by maintaining it at the cellular membrane, thus maintaining its high level of activation, is sufficient to significantly reduce the tumorigenesis," said Kermorgant, who is a lecturer in cellular oncology at the Tumor Biology Centre of Barts Cancer Institute. The team also included a researcher from Cancer Research UK.

Kermorgant said her team now plans to look at preclinical models using actual cancer cell lines.

"In this paper, the cell line that they used was actually a mouse embryonic fibroblast line. It would be more convincing to use a cancer line that exists with the spontaneous MET mutation," said Carl Rountree Jr., assistant professor of pediatrics and pharmacology at the Pennsylvania State University Hershey College of Medicine.

"We will extend this study to other preclinical models to test METdependent tumorigenesis. Especially, we will use human lung and breast cancer cell lines," Kermorgant told SciBX.

Kermorgant said her team also might test endocytosis inhibition in cancers driven by other receptor tyrosine kinases. "We show that one oncogenic receptor did not respond to a specific tyrosine kinase inhibitor but responded to the endocytosis inhibitor," she said. "This could have relevance in the clinic, where resistance against tyrosine kinase inhibitors occurs."

\section{A topical issue}

In addition to testing endocytosis inhibition in other models and cell types, the team hopes to find other compounds that block the process. 
"We used two endocytosis blockers. It is hoped that more compounds will be available in the near future and that we can compare their efficacy," said Kermorgant. "As dynamin has a very broad role in endocytosis, it may be interesting to also consider other targets that more specifically regulate oncogenic receptor endocytosis."

Laurent Audoly, CSO of Pieris AG, said an important next step will be to identify a specific target within the endosomal pathway that is unique for the receptor display and uptake of mutant MET and not critical for housekeeping physiology.

Indeed, researchers contacted by $S c i B X$ said cellular process toxicity will be a primary concern because endocytosis is such a ubiquitous process.

"Inhibiting endocytosis might have many negative side effects due to the inhibition of critical cellular processes depending on constitutive and receptor-induced endocytosis," noted Silvia Giordano, professor of molecular biology at the Institute for Cancer Research and Treatment at the University of Turin Medical School.

Rountree added: "The primary disadvantage of this work is that they didn't address the effects that inhibiting endocytosis has on toxicity. Endocytosis is important for recycling in normal, healthy tissues and it is also important for eliminating phagosomes to clear infection. If we block this process, will patients' healthy tissues be compromised? Will they be more susceptible to infection?"

Shane Olwill, senior director of pharmacology at Pieris, told SciBX that many components of the endocytic pathway might function as tumor suppressors, so inhibiting the entire process in general could have very detrimental effects.

Pieris' PRS-110, an anticalin molecule that antagonizes MET receptor tyrosine kinase by binding the extracellular portion of the receptor rather than the mutation-prone intracellular domain, is in preclinical testing to treat cancer.
"In this paper, the approach was applied topically. This could be an effective strategy to reduce the potentially toxic effects that could result from systemic endocytosis inhibition, but it would really only apply to superficial skin cancers. They would need to find a safe way to administer this systemically," Rountree concluded.

Kermorgant told SciBX her team did not see any toxicity in its mouse experiments.

In addition to treating the subset of patients resistant to tyrosine kinase inhibitors, Kermorgant added that endocytosis inhibition could complement tyrosine kinase inhibitors or MET antibodies in the clinic.

She said the findings are unpatented and unavailable for licensing.

Martz, L. SciBX 4(24); doi:10.1038/scibx.2011.677

Published online June 16, 2011

REFERENCES

1. Joffre, C. et al. Nat. Cell Biol.; published online June 5, 2011; doi:10.1038/ncb2257

Contact: Stéphanie Kermorgant, Queen Mary, University of London, London, U.K. e-mail: s.kermorgant@qmul.ac.uk

2. Lanzetti, L. \& Di Fiore, P.P. Traffic 9, 2011-2021 (2008)

3. Mosesson, Y. et al. Nat. Rev. Cancer 8, 835-850 (2008)

COMPANIES AND INSTITUTIONS MENTIONED

Barts Cancer Institute at Queen Mary, University of London, London, U.K.

Cancer Research UK, London, U.K. Institute for Cancer Research and Treatment at the University of Turin Medical School, Turin, Italy Pennsylvania State University Hershey College of Medicine, Hershey, Pa.

Pfizer Inc. (NYSE:PFE), New York, N.Y.

Pieris AG, Freising-Weihenstephan, Germany

Queen Mary, University of London, London, U.K. 